\title{
A High Side Modes Suppression Dual-Loop Optoelectronic Oscillator With Fabry-Perot Etalon
}

\author{
Kangzhu YIXI*, Fushen CHEN, Chenglong JIANG, and Siyue ZHANG \\ School of Communication and Information Engineering, University of Electronic Science and Technology of China, \\ Chengdu, 611731, China \\ *Corresponding author: Kangzhu YIXI＿E-mail: kangzhu_uestc@163.com
}

\begin{abstract}
A novel dual-loop technique was proposed for single-mode selection in an optoelectronic oscillator (OEO). It consisted of a pump laser and a feedback circuit including an intensity modulator, a Fabry-Perot (FP) etalon, two optical fiber delay lines, two photodetectors, and an amplifier. By inserting the Fabry-Perot etalon, the proposed dual-loop OEO realized a single mode oscillation ranging from $0 \mathrm{~Hz}$ to $20 \mathrm{GHz}$. The strong oscillation mode was present at $15 \mathrm{GHz}$, and the side modes suppression ratio (SMSR) exceeded $140 \mathrm{~dB}$. More over the length of the two fiber loops were just 5 meters and 36 meters.
\end{abstract}

Keywords: Fabry-Perot etalon, optoelectronic oscillator, side modes suppression ratio

Citation: Kangzhu YIXI, Fushen CHEN, Chenglong JIANG, and Siyue ZHANG, "A High Side Modes Suppression Dual-Loop Optoelectronic Oscillator With Fabry-Perot Etalon,” Photonic Sensors, 2014, 4(1): 53-57.

\section{Introduction}

The optoelectronic oscillator (OEO) was first introduced by Yao and Maleki, which used a long fiber to replace the inductance capacitance (LC) resonant circuit in order to reduce the phase noise of the oscillator [1]. The standard OEO structure consists of a pump laser, an intensity modulator, the optical fiber, a photodetector, an amplifier, and a narrow bandpass filter. For the ability to achieve the radio frequency $(\mathrm{RF}) /$ microwave signal with high frequency purity and low phase noise, the OEO is widely used in optical and wireless communications $[2,3]$, radar, and modern instrumentation [4]. It has been proved that the length of the fiber determines the phase noise of the OEO [1]. In particular, it is an effective way to reduce the phase noise by increasing the loop length. However, the mode spacing is inversely proportional to the loop length $[5,6]$. As a result, it is hard to find an electrical filter which is narrow enough to obtain single mode oscillation. The dual-loop OEO with two different length delay lines has solved this tradeoff and attracted considerable attention [7, 8]. The long loop of the structure is used to increase the modes suppression and reduce the phase noise of the system. The short one has a function of increasing the mode spacing which relaxes the requirement of the bandwidth of the electrical filter.

In this work, we designed a novel dual-loop OEO which used a Fabry-Perot (FP) etalon to replace the electrical filter. The FP etalon had high quality, less influenced by the surrounding temperature, and it had lower phase noise than the electrical filter [9]. The transmission and phase characters of the FP etalon were discussed at first.

Received:16 October 2013/ Revised version: 10 December 2013

(C) The Author(s) 2013. This article is published with open access at Springerlink.com DOI: $10.1007 / \mathrm{s} 13320-013-0139-\mathrm{y}$

Article type: Regular 
Then, we analyzed the theory and performance of this novel dual-loop OEO. The simulation result showed that there was only one stable oscillation at the frequency of $15 \mathrm{GHz}$ in the range from $0 \mathrm{~Hz}$ to $20 \mathrm{GHz}$, and the SMSR was over $140 \mathrm{~dB}$.

\section{Principle}

The Fabry-Perot interferometer consists of two parallel high-reflectance glass plates. Light reflects and refracts multiple times between the two plates resulting in the multi-beam interference [10]. In general, for an interferometer, the distance between the two plates may change, but it is a constant for an etalon. The periodic transmission function of the etalon only strengthens the power of special frequencies which are separated by the free spectral range (FSR) of the etalon to oscillate and decreases the power of other frequencies. If the cavity of the FP etalon is filled with air, it can be given that $F S R=c / 2 h$, where $h$ is the length of the cavity. Then, the transmission coefficient of the FP etalon will be expressed as

$$
t(v)=|t(v)| e^{-i \varphi}=\frac{1-R}{1-R \exp (i \varphi)}
$$

where $v$ is the optical frequency, $R$ is the reflection coefficient, and $\varphi(v)=2 \pi v / F S R$ is the round trip phase shift inside the etalon. $\psi(v)$ is the additional optical phase from the etalon which can be written as

$$
\psi(v)=\frac{\varphi(v)}{2}+\tan ^{-1} \frac{R \sin \varphi(v)}{1-R \cos \varphi(v)} .
$$

Figure 1 shows the transmission and phase response characteristics of the FP etalon. In the simulation calculations, we assume the reflection coefficient $R=0.8$, the length of the cavity $h=$ $1.66 \mathrm{~mm}$. It is obvious that the additional optical phase is a function of the input light frequency.

The schematic configuration of the proposed OEO is shown in Fig. 2. The light wave from the laser diode passes through a Mach-Zehnder modulator (MZM) and then is coupled into the FP etalon. After that, the light wave is divided into two optical fiber delay lines with different lengths of 5 meters and 36 meters, respectively. The two loop signals are detected independently by two photodetectors (PD) before the coupler. The output signal after the coupler is amplified and fed back to the electric port of the modulator.
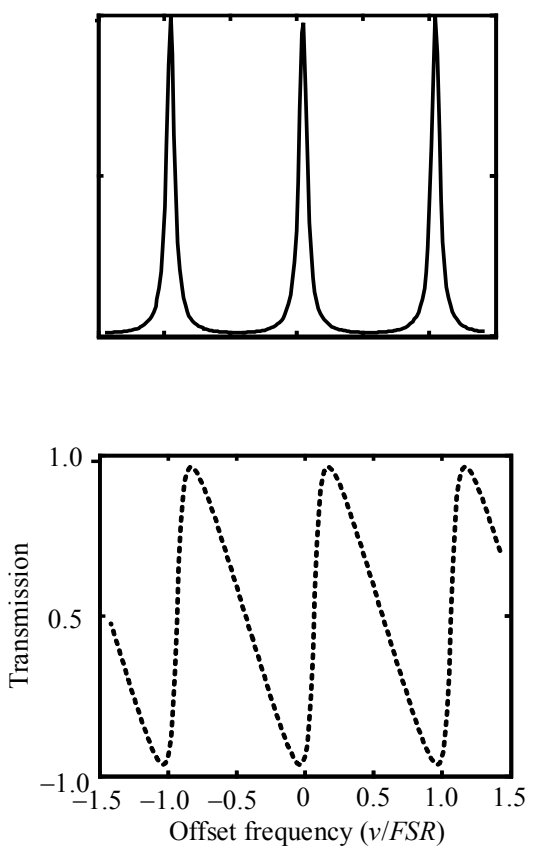

Fig. 1 Transmission and phase response characteristics of the FP etalon.

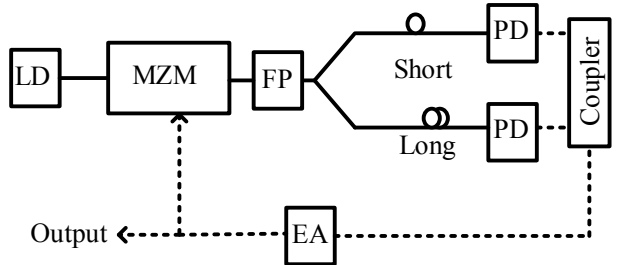

Fig. 2 Schematic of the proposed dual-loop OEO based on the FP etalon.

The incident light can be expressed as $E_{\text {in }}=e_{m} \cos (2 \pi v t)$, and the output optical power from the MZM relates to the applied voltage $V_{\text {in }}=V_{0} \cos \left(2 \pi f_{\text {osc }} t\right)$, where $v$ is the frequency of the laser, and $f_{\text {osc }}$ is the oscillation frequency. The output of the MZM can be given as

$$
\begin{aligned}
E_{\mathrm{MZM}} & =E_{+} \cos \left[2 \pi\left(v-f_{\text {osc }}\right) t\right]+E_{-} \cos (2 \pi v t) \\
& +E_{+} \cos \left[2 \pi\left(v+f_{\text {osc }}\right) t\right]
\end{aligned}
$$

where

$$
E_{+}=-e_{m} J_{1}(a) \sin \left(\pi V_{B} / 2 V_{\pi}\right)
$$

$E_{-}=e_{m} J_{0}\left(\pi V_{0} / 2 V_{\pi}\right) \cos \left(\pi V_{B} / 2 V_{\pi}\right), \quad a=\pi V_{0} / 2 V_{\pi}$, 
$V_{B}$ is the bias voltage, and $V_{\pi}$ is the half-wave voltage.

The modulated optical signal will be coupled into the FP etalon and detected by the PD. The light wave which is exactly in the transmission resonance of the FP etalon will be strengthened, and the others will be filtered. After the optical signals pass through the etalon, for instance, the short loop can be expressed as

$$
E_{\mathrm{FP}}=\frac{\sqrt{2}}{2} \cdot t(v) \cdot E_{\mathrm{MZM}}
$$

In this process, the output of the PD has different kind of frequencies, including three direct current (DC) components. Besides some of the other frequencies are too high to be detected, only two components survive at last as below:

$$
E_{\mathrm{RF}}=\frac{1}{2}|t(v)|^{2} E_{+} E_{-} \rho R \times\left\{\mathrm{co}\left[\mathrm{s} 2 \pi f_{\mathrm{osc}} t-\psi\left(v-f_{\mathrm{osc}}\right)+\psi(v)\right]-\mathrm{co}\left[\mathrm{s} 2 \pi f_{\mathrm{osc}} t+\psi\left(v+f_{\mathrm{osc}}\right)-\psi(v)\right]\right\}
$$

where $R$ is the load impedance of the photodetector, can be simplified as and $\rho$ is responsivity of the detector. Equation (5)

$$
E_{\mathrm{RF}}=\frac{1}{2}|t(v)|^{2} E_{+} E_{-} \rho R \cos \left[\psi(v)-\frac{\psi\left(v+f_{\mathrm{osc}}\right)+\psi\left(v-f_{\mathrm{osc}}\right)}{2}\right] \cos \left[2 \pi f_{\mathrm{osc}} t+\frac{\psi\left(v+f_{\mathrm{osc}}\right)-\psi\left(v-f_{\mathrm{osc}}\right)}{2}\right] .
$$

The power of the oscillation signal of the short loop is

$$
P_{\mathrm{RF}} \propto 1 / \times\left\{1-2 G \cos \left[2 \pi f_{\mathrm{osc}} t+\frac{\psi\left(v+f_{\mathrm{osc}}\right)-\psi\left(v-f_{\mathrm{osc}}\right)}{2}\right]+G^{2}\right\}
$$

where $t$ is the total time delay of the loop, and $G$ is the gain of the loop which includes the gain $G_{A}$ of the amplifier. The self-sustained oscillation needs the feedback loop gain greater than one.

\section{Dual-loop simulations}

For the dual-loop OEO, the short loop and long loop are just as two different cavities which can reduce the numbers of oscillation modes and lower the requirements of the electrical filter. We can use the same method to calculate the dual-loop RF spectrum. The recursive relation can be given as

$$
\begin{gathered}
V_{i}(\omega)=\left(g_{1} e^{j \omega \tau_{1}}+g_{2} e^{j \omega \tau_{2}}\right) V_{i-1}(\omega) \quad \text { (7) Then, the corresponding output pow } \\
P(\omega)=\frac{\left|V_{0}\right|^{2} / 2 R}{1-2\left|g_{1}\right| e^{j \Phi_{1}}-2\left|g_{2}\right| e^{j \Phi_{2}}+\left|g_{1}\right|^{2} e^{j 2 \Phi_{1}}+\left|g_{2}\right|^{2} e^{j 2 \Phi_{2}}+2\left|g_{1}\right|\left|g_{2}\right| e^{j\left(\Phi_{1}+\Phi_{2}\right)}}
\end{gathered}
$$

where $\tau_{1}$ is the short loop delay time, $\tau_{2}$ is the long loop delay time, $\omega$ is the radian frequency of the oscillation frequency, $g_{1}$ is the complex gain of the short loop, and $g_{2}$ is the complex gain of the long loop. The total output voltage is the summation of all circulating fields in the loop. When the oscillation is stable, it can be written as

$$
\begin{aligned}
V_{\text {out }}(\omega) & =\sum_{i=0}^{\infty}\left(g_{1} e^{j \omega \tau_{1}}+g_{2} e^{j \omega \tau_{2}}\right) V_{i-1}(\omega) \\
& =V_{0} /\left[1-\left(g_{1} e^{j \omega \tau_{1}}+g_{2} e^{j \omega \tau_{2}}\right)\right] .
\end{aligned}
$$

Then, the corresponding output power is where $\Phi_{j}=\omega \tau_{j}+\varphi_{j}, \mathrm{j}=1,2$.

In the dual-loop OEO, different lengths of the loops lead to different oscillation modes, and the two loops signals must match the oscillation conditions at the same time:

$$
\begin{gathered}
\Phi_{1}(\omega)=2 k \pi \\
\Phi_{2}(\omega)=2 m \pi \\
\Phi_{1}(\omega)-\Phi_{2}(\omega)=2 k(-m) \pi .
\end{gathered}
$$


$k$ and $m$ are constant, and they are not equal in general. Substituting (10) into (9), we can get the expression of the power of the oscillation frequency:

$$
P(\omega)=\frac{\left|V_{0}\right|^{2} / 2 R}{\left[\left(\left|g_{1}\right|+\left|g_{2}\right|\right)-1\right]^{2}} .
$$

The open loop gain of each loop of the dual-loop oscillation is allowed less than unity, as long as the combined open loop gain of both loops is larger than unity. The oscillation starts from noise, it must satisfy

$$
\left|g_{1}\right|+\left|g_{2}\right|=1 \quad\left|g_{1}\right|=\left|g_{2}\right|=0.5 \text {. }
$$

Equation (12) gives the threshold of the double-loop OEO oscillation. Generally, in the self-oscillation system, the round trip phase of the oscillator has to be modulo integer multiples: $2 \pi N=2 \pi f_{\text {osc }} t+\theta$, where $N$ is an integer, $\theta$ is the additional phase shift caused by the devices in the loop, and $t$ is the delay time of the loop.

The oscillation frequency can be expressed as

$$
\begin{aligned}
f_{\text {osc }} & =\left[2 \pi N-\frac{\psi\left(v+f_{\text {osc }}\right)-\psi\left(v-f_{\text {osc }}\right)}{2}\right] / 2 \pi \tau_{1} \\
& =\left[2 \pi M-\frac{\psi\left(v+f_{\text {osc }}\right)-\psi\left(v-f_{\text {osc }}\right)}{2}\right] / 2 \pi \tau_{2}
\end{aligned}
$$

where $M$ and $N$ are two different integers.

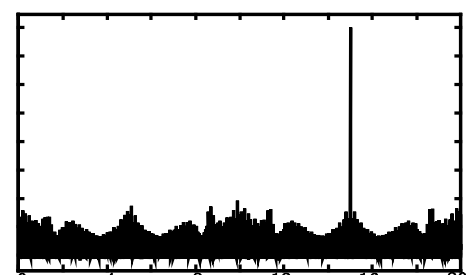

(a)

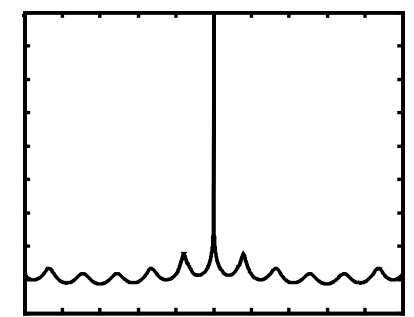

(b)

Fig. 3 Relative power with different frequency spans: (a) $20 \mathrm{GHz}$ and (b) $100 \mathrm{MHz}$.
In the simulation, we assume that the FSR of the FP etalon is $15 \mathrm{GHz}$, and the reflectance $R$ is 0.8 . The wavelength of the incident light is $1550 \mathrm{~nm}$, and the two fiber delay lines are 5 meters and 36 meters long. From Fig. 3(a), the proposed dual-loop OEO demonstrates to be a single mode oscillation between $0 \mathrm{~Hz}$ and $20 \mathrm{GHz}$. The FP etalon can totally take place of the electrical filter in the OEO which agrees with the theory. The strong oscillation mode is present at $15 \mathrm{GHz}$, which is determined by the FSR of the proposed FP etalon in this structure. From Fig. 3(b), we can see the SMSR of the proposed OEO is over $140 \mathrm{~dB}$.

\section{Conclusions}

A dual-loop OEO based on the FP etalon has been proposed and analyzed. The FP etalon can totally take place of the electrical filter in the OEO to achieve the single mode oscillation. The oscillation frequency was $15 \mathrm{GHz}$ determined by the FSR of the proposed FP etalon. By inserting the FP etalon, the length of the OEO became just tens of meters which was much less than that of the traditional dual-loop OEO. And this novel structure can effectively reduce the SMSR. Its $S M S R$ was 50 $\mathrm{dB}$ lower than the single loop OEO with the FP etalon.

Open Access This article is distributed under the terms of the Creative Commons Attribution License which permits any use, distribution, and reproduction in any medium, provided the original author(s) and source are credited.

\section{References}

[1] X. Yao and L. Maleki, "Optoelectronic microwave oscillator," Journal of the Optical Society of America B, 1996, 13(8): 1725-1735.

[2] L. Huo, Y. Dong, C. Lou, and Y. Gao," "Clock extraction using an optoelectronic oscillator from high-speed NRZ signal and NRZ-to-RZ format transformation," Photonics Technology Letters, 2003, 15(7): 981-983.

[3] H. Tsuchida and M. Suzuki, "40-Gb/s optical clock recovery using an injection-locked optoelectronic 
oscillator," Photonics Technology Letters, 2005, 17(1): 211-213.

[4] X. S. Yao and L. Maleki, "Opto-electronic oscillator and its applications," in Proc. SPIE, 1997, 3038: 97-107.

[5] X. S. Yao, L. Maleki, Y. Ji, G. Lutes, and T. Meirong, "Dual-loop opto-electronic oscillator," Frequency Control Symposium, IEEE International, Pasadena, 1998, May 27, pp. 545-549.

[6] T. Zhou and F. Liao, "Principle and experiment analysis of the optoelectronic microwave oscillator," Infrared and Laser Engineering, 2006, 35(z5): 80-83.

[7] L. Maleki, S. Yao, Y. Ji, and V. Ilchenko, "New schemes for improved opto-electronic oscillator," on 1999 Microwave Photonics, Melbourne, Australia,
1999, 1, pp. 177-180.

[8] E. C. Levy, O. Okusaga, M. Horowitz, C. R. Menyuk, W. Zhou, and G. M. Carter, "Comprehensive computational model of single-and dual-loop optoelectronic oscillators with experimental verification," Optics Express, 2010, 18(20): 21461-21476.

[9] J. S. Patel, M. A. Saifi, D. W. Berreman, C. Lin, N. Andreadakis, and S. D. Lee, "Electrically tunable optical filter for infrared wavelength using liquid crystals in a Fabry-Perot etalon," Applied Physics Letters, 1990, 57(17): 1718-1720.

[10] P. D. Atherton, N. K. Reay, J. Ring, and T. R. Hicks, "Tunable Fabry-Perot filters," Optical Engineering, 1981, 20(6): 206805-206805. 